\title{
Prognostic value of the lymphocyte-to-monocyte ratio and other inflammatory markers in malignant pleural mesothelioma
}

\author{
Abdullah Cetin Tanrikulu' ${ }^{1}$ Abdurrahman Abakay' ${ }^{1}$ Halil Komek ${ }^{2} \cdot$ \\ Ozlem Abakay ${ }^{1}$
}

Received: 11 February 2016/Accepted: 29 March 2016/Published online: 11 April 2016

(c) The Japanese Society for Hygiene 2016

\begin{abstract}
Objectives Inflammation plays a role in malignant pleural mesothelioma (MPM) prognosis and symptoms. We investigated the roles of the new and old inflammatory indexes and markers in MPM prognosis.

Methods Two hundred and ninety-two MPM patients (167 male and 125 female) were included in this retrospective study. Demographic parameters were collected from the patients' files. Kaplan-Meier curves and multivariate Cox regression analyses were used for the analysis of prognosis.

Results The mean age of the patients was 58.4 years. The mean survival time was $14.6 \pm 13.0$ months. Twenty-four potential prognostic factors associated with a poor outcome were calculated in the univariate analysis, and 16 potential prognostic factors were associated with a poor prognosis. These 16 potential prognostic factors were also analyzed in multivariate analysis. Multivariate analysis showed that increased age, stage 3-4 disease, the non-epithelial type, a low Karnofsky performance score, a high white blood cell count, and a low lymphocyte-to-monocyte ratio (LMR) were associated with a poor prognosis. The results of the multivariate analysis showed that a decreased LMR was associated with poor survival. Patients with LMR $\leq 2.6 \mathrm{had}$ poor survival compared with those with LMR $>2.6$ (mean 9.6 vs. 17.0 months, respectively; $p=0.004$ ).
\end{abstract}

Abdurrahman Abakay

arahmanabakay@hotmail.com

1 Department of Chest Diseases, Medical School of Dicle University, Diyarbakir, Turkey

2 Department of Nuclear Medicine, Diyarbakir Education and Training Hospital, Diyarbakir, Turkey
Conclusions LMR is an independent marker of prognosis in patients with MPM and is superior to the other inflammation-based markers. The inexpensive nature and easy reproducibility of the hemogram should encourage the use of the LMR in clinical practice.

Keywords Lymphocyte-to-monocyte ratio · Inflammation · Malignant mesothelioma $\cdot$ Prognosis

\section{Introduction}

Malignant pleural mesothelioma (MPM) is a rare aggressive tumor originating from the pleural mesothelium that is associated with poor survival, and there is limited knowledge concerning its natural history [1]. Due to environmental asbestos exposure, MPM is commonly seen in the southeast region of Turkey [2-4]. MPM has a poor prognosis, and the mean survival time has been reported to be approximately 12 months [4-6].

The European Organization for Research and Treatment of Cancer (EORTC) and Cancer and Leukemia Group B (CALGB) devised two prognostic scoring systems for use in patients with mesothelioma [7, 8]. However, these scoring systems are not routinely used for MPM prognosis because they are time-consuming and require costly equipment.

Identification of parameters that are useful, easy, and inexpensive to measure for predicting MPM prognosis is needed. Furthermore, these parameters may be useful for estimating the occurrence of malignant mesothelioma (MM) after asbestos exposure and predicting treatment options and results.

Inflammation plays an important role in the development of MPM. Moreover, during the mesothelioma period, 
patients show signs of increased inflammatory responses such as fever, sweats, and weight loss [9, 10].

Recent studies have identified the neutrophil-lymphocyte ratio (NLR) and the platelet-to-lymphocyte ratio (PLR) as a measure of systemic inflammation; they are relatively inexpensive and readily obtainable reproducible markers that serve as independent prognostic factors in MM patients [3, 11-14]. One study showed that the derived NLR (dNLR) is a prognostic factor for colon cancer [15].

The red cell distribution width (RDW) is a parameter that measures the variability in the size of circulating erythrocytes [16]. It is a routinely measured hemogram. The mean platelet volume (MPV) reflects the platelet size, which is correlated with platelet function and activation. A high MPV predicts platelet activity and the intensity of inflammation [17].

Lymphocytes act as tumor suppressors by inducing cytotoxic cell death and inhibiting tumor cell proliferation and migration [18]. Lymphocyte infiltration into cancer tissue has been associated with a better prognosis in various malignancies [19]. The important role of monocytes and macrophages in cancer, including thoracic cancer, has recently been uncovered [20]. The combined index, the lymphocyte-to-monocyte ratio (LMR), has been demonstrated to be an independent prognostic factor in MPM patients, and the LMR is superior to other inflammationbased prognostic scores [21].

In one study, the dNLR was found to be associated with poor cancer prognosis [22]. This association was not investigated in MPM patients. Zhang et al. [23] showed that the preoperative platelet count and neutrophil-lymphocyte ratio (COP-NLR) can predict the prognosis of patients with lung cancer. In one study, the authors demonstrated that the neutrophil-platelet score (NPS) predicted survival in various common cancers [24].

Based on our knowledge, this is the first study that compares the NPS, COP-NLR, and dNLR, as well as other inflammatory markers, with respect to their effect on MPM prognosis. The aim of this study was to investigate the relationship between potential inflammatory prognostic indicators, such as the NPS, COP-NLR, NLR, dNLR, PLR, MPV, LMR, and RDW, and MPM prognosis.

\section{Materials and methods}

This retrospective study included 338 MPM patients who were diagnosed and treated at Dicle University Hospital between May 2005 and December 2015. The local ethics committee approved the study protocol. Forty-six patients were excluded from the study due to a lack of data and active infection. Two hundred and ninety-two patients were included in the study.
MPM was confirmed by histopathological examination in all of the patients. Histochemical or immunohistochemically staining was used if necessary. Demographic data, asbestos exposure type and time, histopathological subtype of MPM, and basic laboratory parameters were obtained from the patients' files. We used the Butchart staging system in our patients because we could not perform thoracoscopy on all patients [25]. Details on the potential prognostic parameters measured at the time of diagnosis were also obtained from the patients' files.

Approximately half of our MPM patients were received chemotherapy, one of four were received surgical treatments and one of four were received best supportive care treatment.

Blood samples were taken at diagnosis time. Hemogram parameters [including the white blood cell (WBC), hemoglobin, platelet, neutrophil, monocyte and lymphocyte counts] were measured using Cell-Dyn 3700 (Abbott Diagnostics, Lake Forest, IL, USA). The complete blood cell count was measured using a Cell-Dyn 3700 (Abbott Diagnostics).

Assays were performed within $1 \mathrm{~h}$ of collection, after centrifugation at $1500 \mathrm{~g}$ for $10 \mathrm{~min}$ at room temperature of the paired specimens. The inflammatory indexes were defined for MPM patients based on parameters from patients' files that were obtained at the time of diagnosis.

The NLR was determined by dividing the absolute neutrophil count by the absolute lymphocyte count. The PLR was determined by dividing the absolute platelet count by the absolute lymphocyte count. The LMR was determined by dividing the absolute lymphocyte count by the absolute monocyte count. The dNLR neutrophil count was calculated as WBC count - neutrophil count [22].

NPS was defined according to the system proposed by Watt et al. [24]:

NPS 0 neutrophils $\leq 7.5 \times 10^{9} / \mathrm{L}$ and platelets $\leq 400 \times$ $10^{9} / \mathrm{L}$

NPS 1 neutrophils $>7.5 \times 10^{9} / \mathrm{L}$ or platelets $>400 \times$ $10^{9} / \mathrm{L}$

NPS 2 neutrophils $>7.5 \times 10^{9} / \mathrm{L}$ and platelets $>400 \times 10^{9} / \mathrm{L}$

COP-NLR was calculated as follows:

COP-NLR $0 \quad$ NLR $\leq 3$ and platelets $\leq 300 \times 10^{9} / \mathrm{L}$

COP-NLR $1 \quad$ NLR $>3$ or platelets $>300 \times 10^{9} / \mathrm{L}$

COP-NLR 2 NLR $>3$ and platelets $>300 \times 10^{9} / \mathrm{L}$

The following potential prognostic parameters were used, and the mean value of the biochemical parameters was used for prognostic calculations (Table 1). Some of these parameters were age $\geq 60$ and $<60$ years, gender, male or female gender, histopathological subtype, epithelial or non-epithelial, stage 1-2 or 3-4 disease, pleural fluid 
Table 1 Demographic features of mesothelioma patients

\begin{tabular}{|c|c|c|}
\hline Features & $n$ & $\%$ \\
\hline Number of patients & 292 & 100 \\
\hline Mean age of patients & 58.4 & \\
\hline \multicolumn{3}{|l|}{ Gender } \\
\hline Male & 167 & 57.2 \\
\hline Female & 125 & 42.8 \\
\hline History of exposure of asbestos & 174 & 59.6 \\
\hline \multicolumn{3}{|l|}{ Sub-type of MM } \\
\hline Epithelial & 201 & 68.8 \\
\hline Mixed & 16 & 5.6 \\
\hline Unidentified & 67 & 22.9 \\
\hline Sarcomatous & 8 & 2.7 \\
\hline Mean value of WBC $\left(\times 10^{9} / \mathrm{L}\right)(n=292)$ & 9102 & \\
\hline Mean value of neutrophil $\left(\times 10^{9} / \mathrm{L}\right)(n=285)$ & 6.2 & \\
\hline Mean value of lymphocyte $\left(\times 10^{9} / \mathrm{L}\right)(n=285)$ & 1.9 & \\
\hline Mean value of monocyte $\left(\times 10^{9} / \mathrm{L}\right)(n=283)$ & 0.7 & \\
\hline Mean value of platelet $\left(\times 10^{9} / \mathrm{L}\right)(n=292)$ & 340.4 & \\
\hline Mean value of hemoglobin $(\mathrm{g} / \mathrm{L})(n=289)$ & 13.5 & \\
\hline Mean value of sodium $(\mathrm{mmol} / \mathrm{L})(n=273)$ & 138.4 & \\
\hline Mean value of RDW $(\%)(n=285)$ & 15.9 & \\
\hline Mean value of MPV (fL) $(n=284)$ & 8.7 & \\
\hline Mean value of albumin $(\mathrm{g} / \mathrm{dL})(n=278)$ & 3.3 & \\
\hline Mean value of CRP $(\mathrm{mg} / \mathrm{L})(n=208)$ & 46.8 & \\
\hline Mean value of NLR $(n=285)$ & 3.7 & \\
\hline Mean value of PLR $(n=285)$ & 196.0 & \\
\hline Mean value of LMR $(n=283)$ & 5.0 & \\
\hline
\end{tabular}

$R D W$ red cell distribution width, $W B C$ white blood cell, $M P V$ mean platelet volume, PLR platelet-to-lymphocyte ratio, $N L R$ neutrophillymphocyte ratio, $C R P$ C-reactive protein, $L M R$ lymphocyte to monocyte ratio

cytology positive or negative, and Karnofsky performance score (KPS $>60$ or $\leq 60$ ). The NLR was taken as $\leq 3$ and $>3$ from previous studies [3, 14].

Receiver operating characteristic (ROC) curve analysis was performed to select the most appropriate cut-off point for the WBC, neutrophil, lymphocyte, monocyte, albumin, LMR, PLR, RDW, dNLR, MPV, and sodium values to predict poor prognosis in patients with MPM. The score with the maximum sensitivity and specificity was selected as the best cut-off value. Survival results were dichotomized by survival (alive vs. death) in the ROC analysis.

\section{Statistical analysis}

The mean values and standard deviation were calculated for the continuous variables. For continuous variables, we used the independent $t$ test; for categorical variables, we used the chi square test. The duration of survival and the median and mean event times with $95 \%$ confidence intervals were estimated according to the Kaplan-Meier method. The duration of survival was determined as the period between the time of diagnosis and the time of death. If patients were still alive, survival was defined as the period between the times of diagnosis until December 1, 2015.

The proportional hazards regression model with stratification for the clinical trial was used for both the univariate and multivariate analyses. The univariate analyses examined the prognostic importance of all of the aforementioned factors. The Cox proportional hazards model was used to examine the variables. A two-sided test was used, with a 0.05 level of significance. Comparisons of overall survival were made using two-tailed log-rank tests. Only variables with $p$ values $<0.1$ in the univariate analysis were included in the final model for the multivariate analysis.

In the Cox regression analysis, the "backward conditional" method was used. A $p$ value $<0.05$ was considered to indicate statistical significance. In the study group, 32 were alive at the time of this study. Statistical analyses were performed using SPSS statistical program version 15 (SPSS $^{\circledR}$ Inc., Chicago, IL, USA).

\section{Results}

Two hundred and ninety-two MPM patients met the inclusion criteria and were included in this study. The mean age of the MPM patients was $58.4 \pm 12.2(22-87)$ years. One hundred and sixty-seven $(57.2 \%)$ patients were male and $125(42.8 \%)$ were female. Two hundred and one $(68.8 \%)$ patients showed the epithelial-type histopathological subtype (Table 1). The mean survival time was $14.6 \pm 13.0$ months in all of the MPM patients. The mean value of NLR was 3.7, and the mean value of LMR was 5.0 (Table 1).

Twenty-four potential prognostic factors associated with a poor outcome were calculated in the univariate analysis, and 16 potential prognostic factors were associated with a poor prognosis (Table 2). These 16 potential prognostic factors were also analyzed in multivariate analysis.

The results of multivariate analysis showed that an increased age, stage 3-4 disease, the non-epithelial type, a low KPS, a high WBC count, and a low LMR were associated with a poor prognosis. The results of multivariate analysis demonstrated that an LMR $\leq 2.6$ was associated with a poor prognosis of MPM patients. Patients with an LMR $\leq 2.6$ had a 1.8-fold increased mortality rate (Table 2). Figure 1 shows the association between LMR and MPM prognosis. Figure 2 shows the association between dNLR and MPM prognosis. The results of the 
Table 2 Univariate and multivariate analysis of parameters

\begin{tabular}{|c|c|c|c|c|c|c|}
\hline \multirow[t]{2}{*}{ Variable } & \multicolumn{3}{|c|}{ Univariate analysis } & \multicolumn{3}{|c|}{ Multivariate analysis } \\
\hline & $O / N^{*}$ & Survival $^{\mathrm{a}}$ & $p$ & HR & $95 \% \mathrm{CI}$ & $p$ \\
\hline \multicolumn{7}{|c|}{ Age (years) $(n=292)$} \\
\hline$>60$ & $128 / 162$ & 12.1 & $<0.001$ & 1.5 & $(1.1-2.0)$ & 0.011 \\
\hline$\leq 60$ & $120 / 130$ & 16.6 & & & & \\
\hline \multicolumn{7}{|c|}{ Gender $(n=292)$} \\
\hline Male & $145 / 167$ & 13.1 & 0.040 & 1.1 & $(0.8-1.6)$ & 0.45 \\
\hline Female & $103 / 125$ & 16.6 & & & & \\
\hline \multicolumn{7}{|c|}{ Butchart stage $(n=271)$} \\
\hline $1-2$ & $151 / 184$ & 17.1 & 0.000 & 1.7 & $(1.2-2.3)$ & 0.002 \\
\hline $3-4$ & $80 / 87$ & 9.9 & & & & \\
\hline \multicolumn{7}{|c|}{ Pleural fluid cytology $(n=224)$} \\
\hline Negative & $130 / 150$ & 15.6 & 0.60 & & & \\
\hline Positive & $63 / 74$ & 14.0 & & & & \\
\hline \multicolumn{7}{|c|}{ Pathological type $(n=292)$} \\
\hline Epithelial & $167 / 201$ & 13.0 & 0.003 & 1.5 & $(1.1-2.0)$ & 0.011 \\
\hline Others & $81 / 91$ & 12.1 & & & & \\
\hline \multicolumn{7}{|l|}{$\operatorname{KPS}(n=280)$} \\
\hline$\leq 60 \%$ & $48 / 48$ & 9.4 & 0.000 & 2.2 & $(1.5-3.2)$ & $>0.001$ \\
\hline$>60 \%$ & $189 / 232$ & 15.9 & & & & \\
\hline \multicolumn{7}{|c|}{ Platelet count $(n=292)\left(\times 10^{9} / \mathrm{L}\right)$} \\
\hline$\leq 300$ & $111 / 130$ & 15.5 & 0.54 & & & \\
\hline$>300$ & $137 / 162$ & 13.9 & & & & \\
\hline \multicolumn{7}{|c|}{ WBC count $(n=292)\left(\times 10^{9} / \mathrm{L}\right)$} \\
\hline$\leq 8100 \mathrm{~K} / \mathrm{UL}$ & $100 / 120$ & 17.5 & 0.009 & 1.4 & $(0.9-2.3)$ & 0.2 \\
\hline$>8100 \mathrm{~K} / \mathrm{UL}$ & $148 / 172$ & 12.7 & & & & \\
\hline \multicolumn{7}{|c|}{ Hemoglobin $(n=289)(\mathrm{g} / \mathrm{L})$} \\
\hline$\leq 13.2 \mathrm{U} / \mathrm{L}$ & $133 / 151$ & 15.3 & 0.85 & & & \\
\hline$>13.2 \mathrm{U} / \mathrm{L}$ & $112 / 138$ & 13.8 & & & & \\
\hline \multicolumn{7}{|c|}{ Albumin $(n=278)(\mathrm{g} / \mathrm{dL})$} \\
\hline$\leq 3.5$ & $138 / 154$ & 11.9 & 0.000 & 1.3 & $(0.96-1.8)$ & 0.09 \\
\hline$>3.5$ & $101 / 124$ & 17.8 & & & & \\
\hline \multicolumn{7}{|c|}{$\mathrm{CRP}(n=208)(\mathrm{mg} / \mathrm{L})$} \\
\hline$\leq 14.7 \mathrm{U} / \mathrm{L}$ & $44 / 64$ & 13.8 & 0.21 & & & \\
\hline$>14.7 \mathrm{U} / \mathrm{L}$ & $124 / 144$ & 13.9 & & & & \\
\hline \multicolumn{7}{|c|}{$\mathrm{NA}(n=273)(\mathrm{mmol} / \mathrm{L})$} \\
\hline$\leq 138.5$ & $120 / 138$ & 14.5 & 0.68 & & & \\
\hline$>138.5$ & $112 / 135$ & 14.7 & & & & \\
\hline \multicolumn{7}{|l|}{$\operatorname{NLR}(n=285)$} \\
\hline$\leq 3$ & $106 / 127$ & 17.2 & 0.001 & 1.1 & $(0.6-1.9)$ & 0.8 \\
\hline$>3$ & $135 / 158$ & 12.0 & & & & \\
\hline \multicolumn{7}{|c|}{ Derived NLR $(n=285)$} \\
\hline$\leq 1.8$ & $78 / 96$ & 17.1 & 0.01 & 1.2 & $(0.7-1.9)$ & 0.5 \\
\hline$>1.8$ & $163 / 189$ & 13.0 & & & & \\
\hline \multicolumn{7}{|l|}{$\operatorname{PLR}(n=285)$} \\
\hline$\leq 144$ & $83 / 97$ & 17.0 & 0.034 & 1.4 & $(0.9-2.3)$ & 0.1 \\
\hline$>144$ & $158 / 188$ & 12.9 & & & & \\
\hline \multicolumn{7}{|c|}{$\operatorname{MPV}(n=284)(\mathrm{fL})$} \\
\hline$\leq 7.8$ & $110 / 137$ & 14.1 & 0.62 & & & \\
\hline$>7.8$ & $130 / 147$ & 14.6 & & & & \\
\hline
\end{tabular}


Table 2 continued

\begin{tabular}{|c|c|c|c|c|c|c|}
\hline \multirow[t]{2}{*}{ Variable } & \multicolumn{3}{|c|}{ Univariate analysis } & \multicolumn{3}{|c|}{ Multivariate analysis } \\
\hline & $O / N^{*}$ & Survival $^{\mathrm{a}}$ & $p$ & HR & $95 \% \mathrm{CI}$ & $p$ \\
\hline \multicolumn{7}{|c|}{$\operatorname{RDW}(n=285)(\%)$} \\
\hline$\leq 14.6$ & $78 / 102$ & 14.6 & 0.13 & & & \\
\hline$>14.6$ & $163 / 183$ & 14.2 & & & & \\
\hline \multicolumn{7}{|c|}{ Neutrophil count $(n=285)\left(\times 10^{9} / \mathrm{L}\right)$} \\
\hline$\leq 5.3$ & $101 / 122$ & 16.8 & 0.015 & 1.4 & $(0.8-1.7)$ & 0.2 \\
\hline$>5.3$ & $140 / 163$ & 12.4 & & & & \\
\hline \multicolumn{7}{|c|}{ Lymphocyte count $(n=285)\left(\times 10^{9} / \mathrm{L}\right)$} \\
\hline$\leq 2.0$ & $134 / 158$ & 13.2 & 0.094 & & & \\
\hline$>2.0$ & $107 / 127$ & 15.8 & & & & \\
\hline \multicolumn{7}{|c|}{ Monocyte count $(n=283)\left(\times 10^{9} / \mathrm{L}\right)$} \\
\hline$\leq 0.55$ & $94 / 116$ & 16.6 & 0.015 & 1.2 & $(0.8-1.7)$ & 0.3 \\
\hline$>0.55$ & $145 / 167$ & 12.7 & & & & \\
\hline \multicolumn{7}{|c|}{ LMR $(n=283)$} \\
\hline$\leq 2.6$ & $91 / 104$ & 9.6 & 0.000 & 1.8 & $(1.2-2.7)$ & 0.004 \\
\hline$>2.6$ & $148 / 179$ & 17.0 & & & & \\
\hline \multicolumn{7}{|c|}{ CRP/albumin $(n=201)$} \\
\hline$\leq 7.5$ & $67 / 89$ & 13.7 & 0.48 & & & \\
\hline$>7.5$ & $97 / 112$ & 14.1 & & & & \\
\hline \multicolumn{7}{|c|}{ Combine platelet NLR $(n=285)$} \\
\hline 0 & $53 / 59$ & 17.8 & 0.006 & 1.3 & $(0.6-2.7)$ & 0.5 \\
\hline 1 & $110 / 138$ & 15.0 & & & & \\
\hline 2 & $78 / 88$ & 11.0 & & & & \\
\hline \multicolumn{7}{|c|}{ NPS $(n=285)$} \\
\hline 0 & $134 / 162$ & 16.4 & 0.05 & 1.7 & $(0.9-3.3)$ & 0.1 \\
\hline 1 & $87 / 100$ & 11.6 & & & & \\
\hline 2 & $20 / 23$ & 11.7 & & & & \\
\hline
\end{tabular}

Bold values indicate better results

$O$ observed death number, $N$ total patient number, $H R$ hazard ratio, $R D W$ red cell distribution width, $M P V$ mean platelet volume, $P L R$ platelet-to-lymphocyte ratio, $N L R$ neutrophil-lymphocyte ratio, $W B C$ white blood cell, $C R P$ C-reactive protein, $L M R$ lymphocyte to monocyte ratio, NA sodium, KPS Karnofsky performance status, NPS the neutrophil-platelet score

${ }^{\text {a }}$ Mean survival (months)

multivariate analysis demonstrated a more significant relationship between LMR and poor MPM prognosis than other inflammatory parameters (NLR, PLR, dNLR, NPS and COP-NLR).

\section{Discussion}

Previous studies focused on developing a parameter that could accurately predict MPM prognosis. Several models were defined, using measurements that included: symptoms, pathologic factors, stage and some blood parameters; unfortunately, none were considered to be ideal.

EORTC and CALGB analyzed large numbers of patients enrolled in MPM studies and identified the following poor prognostic factors for MM [7, 8]: non- epithelioid histology, bad performance status, presence of chest pain, age older than 75 years, male gender, WBC $8.3 \times 10^{9} / \mathrm{L}$ or greater, platelet number over $400,000 / \mu \mathrm{L}$, and an LDH level $>500$ IU/L.

Similarly, we found that the prognostic factors of increased age, stage 3-4 disease, the non-epithelial type, a low KPS, and a high WBC count were associated with a poor survival.

In our previous study, our results indicated that pleural fluid cytology was not associated with mesothelioma prognosis [4]. In the present study, pleural fluid cytology was associated with mesothelioma prognosis in univariate but not in multivariate analysis.

Hypoalbuminemia is associated with poor prognosis of MM. In one study, albumin $<3.5 \mathrm{~g} / \mathrm{L}$ was independently associated with a decrease in survival [26]. PLR was 


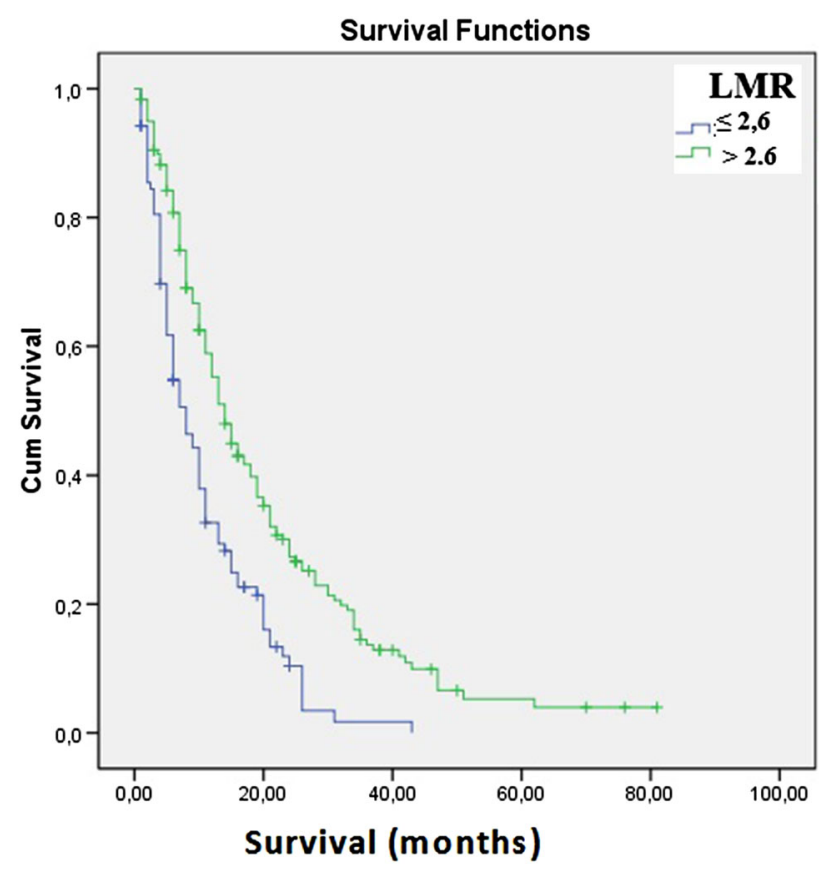

Fig. 1 Kaplan-Meier survival curves according to LMR $(p<0.001)$ $(n=283)$

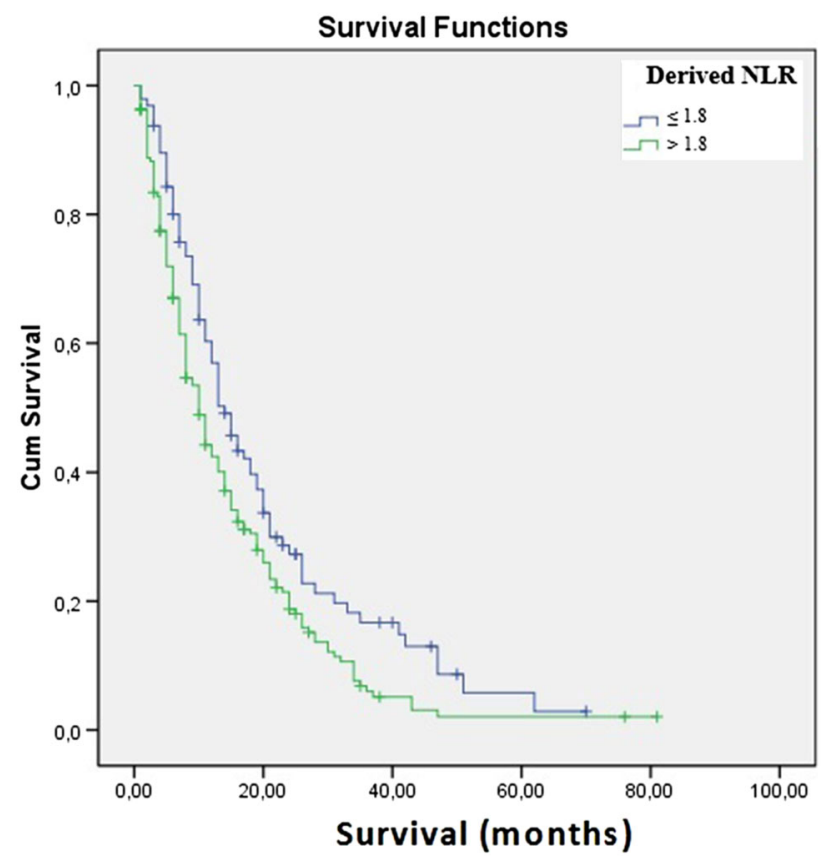

Fig. 2 Kaplan-Meier survival curves according to dNLR $(p=0.01)$ $(n=285)$

associated with poor prognosis [13]. We found that hypoalbuminemia and high PLR were associated with a poor prognosis in univariate analysis but not in multivariate analysis.
The pathological role of chronic inflammation in the development of MPM is already known [27]. Based on our results, inflammatory-based biomarkers may be predictive of survival, and the AMI may be viewed as an inexpensive predictor of MM prognosis at the time of diagnosis.

Many investigations have presented the role of inflammatory parameters such as the NLR and PLR in the prognosis of MPM [11-13, 28]. These studies have reported that the mortality is increased patients with an NLR $\geq 3[12,28]$. One study showed that dNLR is a prognostic factor for colon cancer [15]. The dNLR was not studied in MPM patients. In univariate analysis, we found that the NLR and dNLR were associated with poor survival in MPM patients. A possible explanation for this finding is that MPM patients with more advanced disease at the time of diagnosis may have a more excessive systemic inflammatory response and therefore a higher NLR and dNLR.

The newly investigated inflammatory marker COP-NLR was capable of predicting the prognosis of patients with lung cancer [23]. In another study, the authors demonstrated that NPS predicted survival in various common cancers [24]. These two inflammatory prognostic scores included a high platelet level and were not studied in mesothelioma patients. Platelets can secrete cytokines and growth factors, such as vascular endothelial growth factor, platelet-derived growth factor, TGF- $\beta$, and FGF [29, 30], which, in turn, contribute to cancer progression, including angiogenesis, cell migration and proliferation, and the epithelial to mesenchymal transition [31]. In many studies, a similar high platelet count was associated with mesothelioma prognosis $[4,7,8]$. In this study, the platelet count was not significant. However, the COP-NLR and NPS were associated with MPM prognosis in univariate analysis.

The LMR was found to be an independent prognostic marker for survival in patients with MPM, and the LMR is superior to other inflammation-based prognostic scores [21]. In this study, patients with an LMR $<2.74$ exhibited a median survival was 5.0 months, while those with an LMR $\geq 2.74$ had a median survival of 14.0 months $(p=0.000)$ [21]. Also, in this study, the NLR and PLR were not associated with MPM prognosis in multivariate analysis [21]. Similarly, we found that the LMR was the only associated inflammatory prognostic factor in multivariate analysis. The LMR was also demonstrated to be an independent predictor of survival in various patients with lung cancer [32].

A potential limitation of this study is that it is a retrospective and single-center study. Additionally, the treatment regimens and outcomes were not investigated in this study. However, this investigation demonstrated that the LMR is an independent marker of prognosis in patients 
with MPM and is superior to the other inflammation-based markers (e.g., NLR, dNLR, NPS, and COP-NLR).

LMR is easily assessed using a simple complete blood count test and is both technically and financially feasible to predict the patients' clinical outcomes in routine practice. The LMR is a useful marker, given the high prevalence and the prognostic importance of increased levels. The inexpensive nature and easy reproducibility of the hemogram should encourage its use in clinical practice. Despite our findings, this index must be validated in a large, prospective study.

Acknowledgments The research was not financially supported.

\section{Compliance with ethical standards}

Conflict of interest The authors declare that they have no conflict of interest.

Human and animal rights All procedures performed in studies involving human participants were in accordance with the ethical standards of the institutional research committee and with the 1964 Helsinki Declaration and its later amendments or comparable ethical standards. Dicle University Ethics Committee obtained ethical approval.

Informed consent Informed consent was obtained from all individual participants included in the study.

\section{References}

1. Szychlinska MA, Parenti R, Loreto C, et al. Fluoro edenite-associated pathogenesis in pleural malignant mesothelioma. Acta Medica Mediterranea. 2014;30:981-9.

2. Tanrikulu AC, Senyigit A, Dagli CE, et al. Environmental malignant pleural mesothelioma in Southeast Turkey. Saudi Med J. 2006;27:1605-7.

3. Abakay O, Tanrikulu AC, Palanci Y, Abakay A. The value of inflammatory parameters in the prognosis of malignant mesothelioma. J Int Med Res. 2014;42(2):554-65.

4. Tanrikulu AC, Abakay A, Kaplan MA, et al. A clinical, radiographic and laboratory evaluation of prognostic factors in 363 patients with malignant pleural mesothelioma. Respiration. 2010;80:480-7.

5. Vogelzang NJ. Malignant mesothelioma: diagnostic and management strategies for 1992. Semin Oncol. 1992;19:64-71.

6. Schouwink H, Korse CM, Bonfrer JM, et al. Prognostic value of the serum tumour markers Cyfra 21-1 and tissue polypeptide antigen in malignant mesothelioma. Lung Cancer. 1999;25:25-32.

7. Curran D, Sahmoud T, Therasse P, et al. Prognostic factors in patients with pleural mesothelioma: the European Organization for Research and Treatment of Cancer experience. J Clin Oncol. 1998;16:145-52.

8. Herndon JE, Green MR, Chahinian AP, et al. Factors predictive of survival among 337 patients with mesothelioma treated between 1984 and 1994 by the cancer and Leukemia Group B. Chest. 1998;113:723-31.

9. Nowak AK, Stockler MR, Byrne MJ. Assessing quality of life during chemotherapy for pleural mesothelioma: feasibility, validity, and results of using the European Organization for
Research and Treatment of Cancer core quality of life questionnaire and lung cancer module. J Clin Oncol. 2004;22:3172-80.

10. Robinson BW, Musk AW, Lake RA. Malignant mesothelioma. Lancet. 2005;366:397-408.

11. Kao SC, Pavlakis N, Harvie R, et al. High blood neutrophil-tolymphocyte ratio is an indicator of poor prognosis in malignant mesothelioma patients undergoing systemic therapy. Clin Cancer Res. 2010;16:5805-13.

12. Kao SC, Vardy J, Chatfield M, et al. Validation of prognostic factors in malignant pleural mesothelioma: a retrospective analysis of data from patients seeking compensation from the New South Wales Dust Diseases Board. Clin Lung Cancer. 2013;14:70-7.

13. Pinato DJ, Mauri FA, Ramakrishnan R, et al. Inflammation-based prognostic indices in malignant pleural mesothelioma. J Thorac Oncol. 2012;7:587-94.

14. Tanrikulu AC, Hocanli I, Yilmaz A, et al. The value of new inflammatory parameters in malignant mesothelioma prognosis. Acta Medica Mediterranea. 2015;31:9-15.

15. Absenger G, Szkandera J, Pichler M, et al. A derived neutrophil to lymphocyte ratio predicts clinical outcome in stage II and III colon cancer patients. Br J Cancer. 2013;23(109):395-400.

16. Schweiger DJ. Red cell distribution width in sickle cell anemia. Am J Med Technol. 1981;47:231-3.

17. Bath PM, Butterworth RJ. Platelet size: measurement, physiology and vascular disease. Blood Coagul Fibrinolysis. 1996;7:157-61.

18. Coussens LM, Werb Z. Inflammation and cancer. Nature. 2002;42:806-67.

19. De Giorgi U, Mego M, Scarpi E, et al. Relationship between lymphocytopenia and circulating tumor cells as prognostic factors for overall survival in metastatic breast cancer. Clin Breast Cancer. 2012;12(4):264-9.

20. Lievense LA, Bezemer K, Aerts JG, Hegmans JP. Tumor-associated macrophages in thoracic malignancies. Lung Cancer. 2013;80:256-62.

21. Yamagishi T, Fujimoto N, Nishi H, et al. Prognostic significance of the lymphocyte-to-monocyte ratio in patients with malignant pleural mesothelioma. Lung Cancer. 2015;90(1):111-7.

22. Proctor MJ, McMillan DC, Morrison DS, et al. A derived neutrophil to lymphocyte ratio predicts survival in patients with cancer. Br J Cancer. 2012;107(4):695-9.

23. Zhang $\mathrm{H}$, Zhang L, Zhu K, et al. Prognostic significance of combination of preoperative platelet count and neutrophil-lymphocyte ratio (COP-NLR) in patients with non-small cell lung cancer: based on a large cohort study. PLoS One. 2015;10(5):e0126496.

24. Watt DG, Proctor MJ, Park JH, et al. The neutrophil-platelet score (NPS) predicts survival in primary operable colorectal cancer and a variety of common cancers. PLoS One. 2015;10(11):e0142159.

25. Butchart EG, Ashcroft T, Barnsley WC, Holden MP. Pleuropneumonectomy in the management of diffuse malignant mesothelioma of the pleura. Experience with 29 patients. Thorax. 1976;31:15-24.

26. Pilling JE, Dusmet ME, Ladas G, Goldstraw P. Prognostic factors for survival after surgical palliation of malignant pleural effusion. J Thorac Oncol. 2010;5:1544-50.

27. Hillegass JM, Shukla A, Lathrop SA, et al. Inflammation precedes the development of human malignant mesotheliomas in a SCID mouse xenograft model. Ann $N$ Y Acad Sci. 2010;1203:7-14.

28. Kao SC, Klebe S, Henderson DW, et al. Low calretinin expression and high neutrophil-to-lymphocyte ratio are poor prognostic factors in patients with malignant mesothelioma undergoing extrapleural pneumonectomy. J Thorac Oncol. 2011;6:1923-9. 
29. Banks RE, Forbes MA, Kinsey SE, et al. Release of the angiogenic cytokine vascular endothelial growth factor (VEGF) from platelets: significance for VEGF measurements and cancer biology. Br J Cancer. 1998;77:956-64.

30. Harrison P, Cramer EM. Platelet alpha-granules. Blood Rev. 1993;7:52-62.

31. Guo F, Parker Kerrigan BC, Yang D, et al. Post-transcriptional regulatory network of epithelial-to-mesenchymal and mesenchymal-to-epithelial transitions. J Hematol Oncol. 2014;5(7):19.

32. Hu P, Shen H, Wang G, et al. Prognostic significance of systemic inflammation-based lymphocyte-monocyte ratio in patients with lung cancer: based on a large cohort study. PLoS One. 2014;9:e108062. 